\title{
Cestodos de quirópteros del Parque Nacional Cerros de Amotape, Tumbes, Perú
}

Cestodes of bats from the National Park Cerros de Amotape, Tumbes, Peru

\author{
Marina Vargas C. ${ }^{1}$, Rosa Martínez R. $^{2}$ y Manuel Tantaleán V. ${ }^{3}$
}

\begin{abstract}
1 Facultad de Ciencias Biológicas, Universidad Nacional Mayor de San Marcos. Lima, Perú. E-mail Marina Vargas: marina_vc243@ hotmail.com

2 Laboratorio de Parasitología de Fauna Silvestre. Facultad de Ciencias Biológicas, Universidad Nacional Mayor de San Marcos. Apartado 110058, Lima 11, Perú. E-mail Rosa Martínez: rmartinezr@ E-mail Rosa Matin

3 Laboratorio de Parasitología, Facultad de Medicina Veterinaria. Universidad Peruana Cayetano Heredia. Lima, Perú. E-mail Manuel Tantaleán: mtantaleanv@ hotmail.com
\end{abstract}

Presentado: $13 / 04 / 2009$ Aceptado: 07/05/2009 Publicado online: 28/08/2009

\section{Resumen}

En septiembre 2006, en el Parque Nacional Cerros de Amotape, departamento de Tumbes, Perú; 39 individuos de murciélagos, pertenecientes a 16 especies fueron capturados. El análisis parasitológico determino que solo dos individuos de las especies Phyllostomus hastatus (Phyllostomidae) y Noctilio leporinus (Noctilionidae) estaban parasitados. Los cestodos fueron colectados del intestino delgado e identificados como Atriotaenia hastati Vaucher, 1982 (Anoplocephalidae) y Vampirolepis sp. (Hymenolepididae). Atriotaenia hastati es un nuevo registro para el Perú y Vampirolepis sp. es registrado por primera vez en Tumbes y en un nuevo huésped, Noctilio leporinus.

Palabra clave: Atriotaenia hastati, Vampirolepis, Phyllostomus hastatus, Noctilio leporinus, Tumbes.

\section{Abstract}

In September 2006, at Parque Nacional Cerros de Amotape, department of Tumbes, Peru, 39 individuals of bats belonging to 16 species were captured. Parasitological analysis determined that only two individuals of the species Phyllostomus hastatus (Phyllostomidae) and Noctilio leporinus (Noctilionidae) were parasitized. The cestodes were collected from the small intestine and identified as Atriotaenia hastati Vaucher, 1982 (Anoplocephalidae) and Vampirolepis sp. (Hymenolepididae). Atriotaenia hastati is a new record for Peru and Vampirolepis sp. is registered for the first time in Tumbes and a new host, Noctilio leporinus.

Keywords: Atriotaenia hastati, Vampirolepis, Phyllostomus hastatus, Noctilio leporinus, Tumbes

\section{Introducción}

El Perú, es uno de los países que presenta una gran diversidad de quirópteros a nivel del mundo, ocupando el segundo lugar en el neotrópico con 8 familias, 61 géneros, y más de 160 especies (Pacheco et al. 2007, Pacheco 2008). A pesar de esta gran diversidad en nuestro país se han realizado pocos estudios relacionados con la fauna parasitaria de los quirópteros.

En el Perú los trabajos sobre cestodos en murciélagos son escasos. Vaucher (1986) fue uno de los primeros en estudiarlos, registró una nueva especie, Hymenolepis mazanensis, en Saccopteryx bilineata y Rhinchonycteris naso, huéspedes procedentes del departamento de Loreto; posteriormente, Mendoza et al. (1997) registraron a Vampirolepis artibei y Vampirolepis sp. procedentes de Ica.

El presente trabajo describe los cestodos que parasitan los murciélagos colectados en una evaluación de biodiversidad en la zona del Parque Nacional Cerros de Amotape.

\section{Material y métodos}

En septiembre del 2006 (época seca) se capturaron un total de 39 quirópteros en el Parque Nacional Cerros de Amotape, en dos localidades: (1) Faical, (0349'19”S, 80²3'15”W) a $350 \mathrm{~m}$ de altitud, donde se ubicaron 4 zonas de muestreo: (a) Quebrada facial, (b) Estación Biológica facial, (c) Quebrada las Pavas y (d) Quebrada la Unión. Corresponden a un bosque de transición entre el bosque tropical del pacífico y el bosque

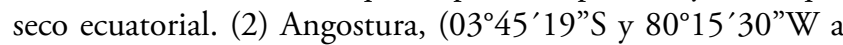
$74 \mathrm{~m}$ de altitud, donde se ubicaron dos zonas de muestreo: (e) Quebrada Angostura y (f) Angostura Platanal. Corresponden al bosque seco ecuatorial, (Pacheco et al.2007).

Los quirópteros fueron capturados utilizando diez redes de niebla de 12 × 2,6 m, colocadas durante 3 días en cada localidad de muestreo, considerando la ruta de vuelo, posibles refugios y áreas de forrajeo; la métodología empleada se detalla en Simmons \& Voss (1998), Hice et al. (2004) y Pacheco et al. (2007). Las especies capturadas fueron inicialmente identificadas en la zona de muestreo y posteriormente confirmadas en el Departamento de Mastozoología del Museo de Historia Natural de la Universidad Nacional Mayor de San Marcos.

Los cestodos fueron aislados del tracto intestinal y fijados en formol al 10\%, posteriormente coloreados con carmín acético de Semichon o carmín clorhídrico, previamente fueron colocados en acido acético al $1 \%$ por un tiempo de 3 a 12 horas para eliminar los corpúsculos calcáreos.

Las mediciones se realizaron utilizando un ocular micrométrico calibrado; las medidas se expresan en milímetros, anotando primero el promedio y luego el rango entre paréntesis; los dibujos se hicieron con una cámara lúcida.

\section{Resultados}

De los 39 quirópteros, sólo Phyllostomus hastatus (1/1) y Noctilio leporinus (1/1) se encontraron parasitados con cestodos pertenecientes a las familias Anoplocephalidae e Hymenolepididae (Tabla 1). Seguidamente cada especie es descrita por ser nuevos registros para el Perú.

\section{Familia Anoplocephalidae}

\section{Atriotaenia hastati Vaucher, 1982}

$$
\text { (Figs.1-7) }
$$

Descripción: Basada en 10 especímenes de un total aproximado de 70 individuos.

Longitud total del estróbilo 21 (17-28). El escolex, desprovisto de ganchos, tiene una longitud de $0,4(0,35-0,49)$ por $0,493(0,3-0,64)$ de ancho. Las ventosas poseen un diámetro de $0,139(0,11-0,15)$. El número de proglótidos maduros es de 6 y el de grávidos de 3 o 4, con un solo juego de aparato reproductor. Proglótidos ligeramente craspedotes; los maduros miden de $0,893(0,75-1,4)$ de largo por $1,47(1,22-1,98)$ de ancho; poros genitales irregularmente alternos. El atrio genital 
Tabla. 1. Especies de quirópteros colectados y cestodos encontrados en las localidades de Faical y Angostura del Parque Nacional Cerros de Amotape (septiembre 2006).

\begin{tabular}{llll}
\hline \multicolumn{2}{c}{ Quirópteros colectados } & \multirow{2}{*}{ Cestodos } \\
\cline { 1 - 2 } Especie & Ind. & \\
Phyllostomidae & & \\
1. Lophostoma silvicolum & 5 & \\
2. Carollia brevicauda & 1 & \\
3. Carollia perspicillata & 1 & \\
4. Platyrrhinus matapalensis & 1 & \\
5. Artibeus jamaicensis & 7 & \\
6. Artibeus fraterculus & 6 & \\
7. Vampyrum spectrum & 1 & \\
8. Sturnira luisi & 3 & \\
9. Myotis riparius & 1 & \\
10. Glossophaga soricina & 2 & \\
11. Desmodus rotundus & 2 & \\
12. Diaemus youngi & 1 & \\
13. Phyllostomus discolor & 5 & \\
14. Phyllostomus hastatus & 1 & Atriotaenia hastati \\
Vespertilionidae & & \\
15. Eptesicus chiriquinus & 1 & \\
Noctilionidae & & \\
16. Noctilio leporinus & 1 & Vampirolepis sp. \\
\hline
\end{tabular}

(1)

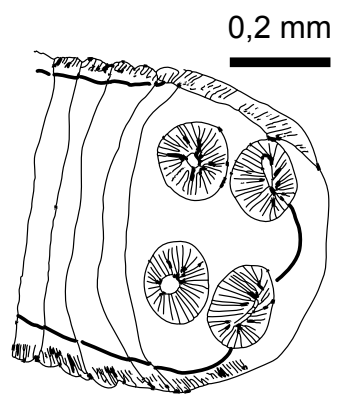

(2)

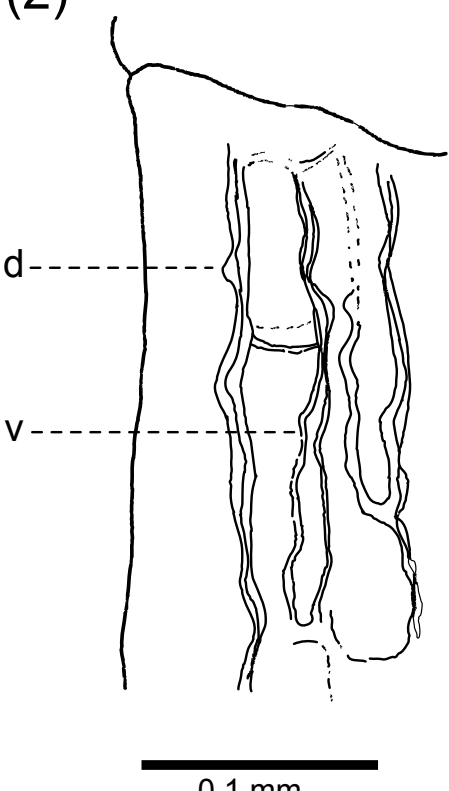

(3)
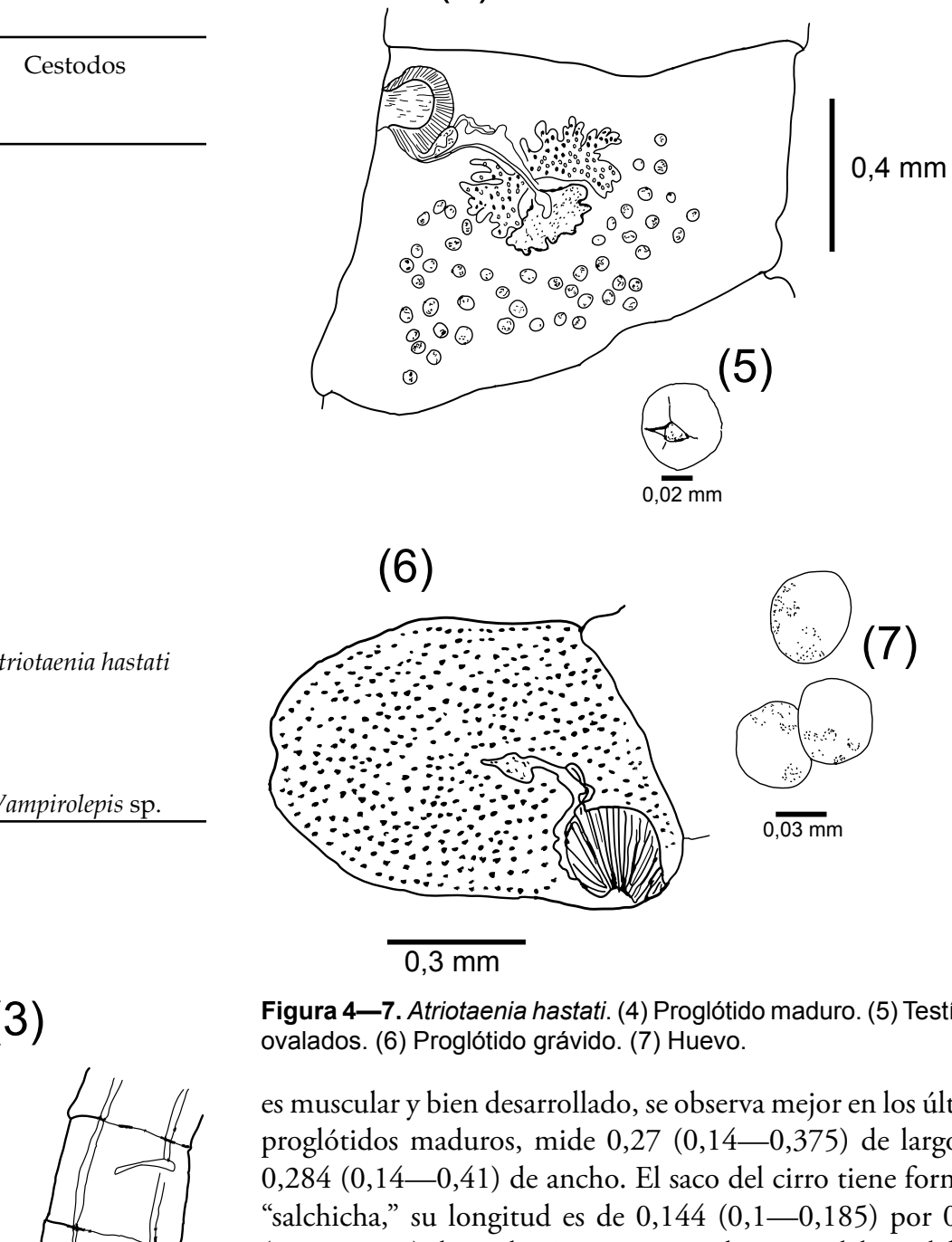

(5)

(6)

\section{$0,3 \mathrm{~mm}$}

Figura 4-7. Atriotaenia hastati. (4) Proglótido maduro. (5) Testículos ovalados. (6) Proglótido grávido. (7) Huevo.

es muscular y bien desarrollado, se observa mejor en los últimos proglótidos maduros, mide $0,27(0,14-0,375)$ de largo por $0,284(0,14-0,41)$ de ancho. El saco del cirro tiene forma de "salchicha," su longitud es de $0,144(0,1-0,185)$ por 0,039 $(0,02-0,07)$ de ancho. La vagina se ubica por debajo del saco del cirro, durante su recorrido se expande gradualmente hasta formar el receptáculo seminal el cual se encuentra situado a nivel del borde anterior de las glándulas vitelógenas, en la parte posterior del ovario, el cual es bilobulado. No se pudo distinguir el útero. Los testículos son numerosos, generalmente agrupados, en número de 53 a 60 y en algunos casos hasta 70; la localización de estos es posterior y lateral al ovario (hasta su parte media) y a las glándulas vitelógenas. Los proglótidos grávidos son más largos que anchos y miden $1,9(1,42-2,35)$ de diámetro. Huevos de $0,046(0,03-0,068)$ de diámetro.

Huésped: Phyllostomus hastatus (Fam. Phyllostomidae).

Localización: En todo el tracto intestinal, formando nódulos.

Localidad: Angostura. Distrito Pampas de Hospital. Provincia de Tumbes.

Material: Depositado en la Colección Helmintológica del laboratorio de Parasitología de Fauna Silvestre. Facultad de Ciencias Biológicas, Universidad Nacional Mayor de San Marcos.

\section{Familia Hymenolepididae}

Vampirolepis sp.

(Figs.8-10)
Figura 1-3. Atriotaenia hastati. (1) Vista apical del escolex. (2) Proglótido mostrando la ramificación de los conductos excretores. d: canal excretor dorsal y v: canal excretor ventral. (3) Proglótido inmaduro, mostrando la disposición irregular de los poros genitales. 
Descripción: Basada en 3 especímenes.

De pequeña longitud, miden 3,28 (3,15-3,45) de largo por $0,16(0,15-0,2)$ de ancho. Escolex grande y bien notorio, de $0,458(0,375-0,6)$ de largo por $0,4(0,37-0,45)$ de ancho, provisto de cuatro fuertes ventosas de $0,091(0,07-0,102)$ de diámetro. Rostelo armado de 48 a 50 ganchos del tipo fraternoide, con una longitud de $0,028(0,025-0,032)$, mango ligeramente curvado, guarda mas grande que la hoja y de contextura ligeramente gruesa al inicio adelgazándose a medida que se acerca a su extremo final el que termina en punta roma; la hoja, a comparación del mango y la guarda, es corta, curvada y puntiaguda. Cuello largo 0,886 $(0,86-0,9)$ por $0,173(0,17-0,18)$. Proglótidos ligeramente craspedotes, más anchos que largos y en maduración progresiva, miden 0,075 de largo por 0,162 de ancho. Con tres testículos ovalados, dispuestos transversalmente en la parte posterior del proglótido.

Huésped: Noctilio leporinus (Fam. Noctilionidae).

Localización: Intestino.

Localidad: Quebrada Faical. Distrito Pampas de Hospital. Provincia de Tumbes

Especímenes: Depositado en la Colección Helmintológica del laboratorio de Parasitología de Fauna Silvestre. Facultad de Ciencias Biológicas, Universidad Nacional Mayor de San Marcos.

\section{Discusión}

El género Atriotaenia fue creado por Sandground, 1926 para cestodos encontrados en Nasua nasua (Atriotaenia sandgroundi); fue considerado sinónimo de Oochoristica (parásito de reptiles, carnívoros insectívoros, marsupiales, primates y quirópteros) y de Mathevotaenia Akumyan, 1976 por Della Santa (1956), pero Schmidt (1986) los diferencia de la siguiente manera: en Oochoristica los proglótidos son de tipo acraspedote y en Mathevotaenia son craspedote y sin receptáculo seminal, en cambio, en Atriotaenia los proglótidos son craspedotes y poseen receptáculo seminal, además de un atrio genital bien desarrollado y un sistema excretor con finos canales asociados a los conductos ventrales que a su vez se comunican a los troncos principales.

Atriotaenia hastati, fue descrita por Vaucher (1982) de Phyllostomus hastatus, capturado en Paraguay y es la única especie de Atriotaenia que parasita a quirópteros; esta especie se caracteriza por el atrio genital voluminoso que se observa mejor en los últimos proglótidos maduros e inicio de los grávidos, el tipo de ovario (bilobulado), posición de la vagina posterior al saco del cirro y número de testículos, entre 50-60. Todos éstos detalles se observan en nuestros especímenes, aunque en algunos proglótidos hemos podido contar hasta 70 testículos, lo que consideramos como una variación intraespecífica. Este es el primer registro de Atriotaenia hastati en el Perú.

Finalmente, en Noctilio leporinus encontramos cestodos que identificamos como Vampirolepis sp. por tener 3 testículos en la parte posterior del proglótido y por el número y morfología de los ganchos. No se pudo determinar la especie debido a que los proglótidos se encontraban en proceso de maduración, por lo que fue imposible observar con claridad otros caracteres como el tamaño del saco del cirro, presencia de espinas en el cirro, forma del ovario, etc.; sin embargo, fue posible establecer

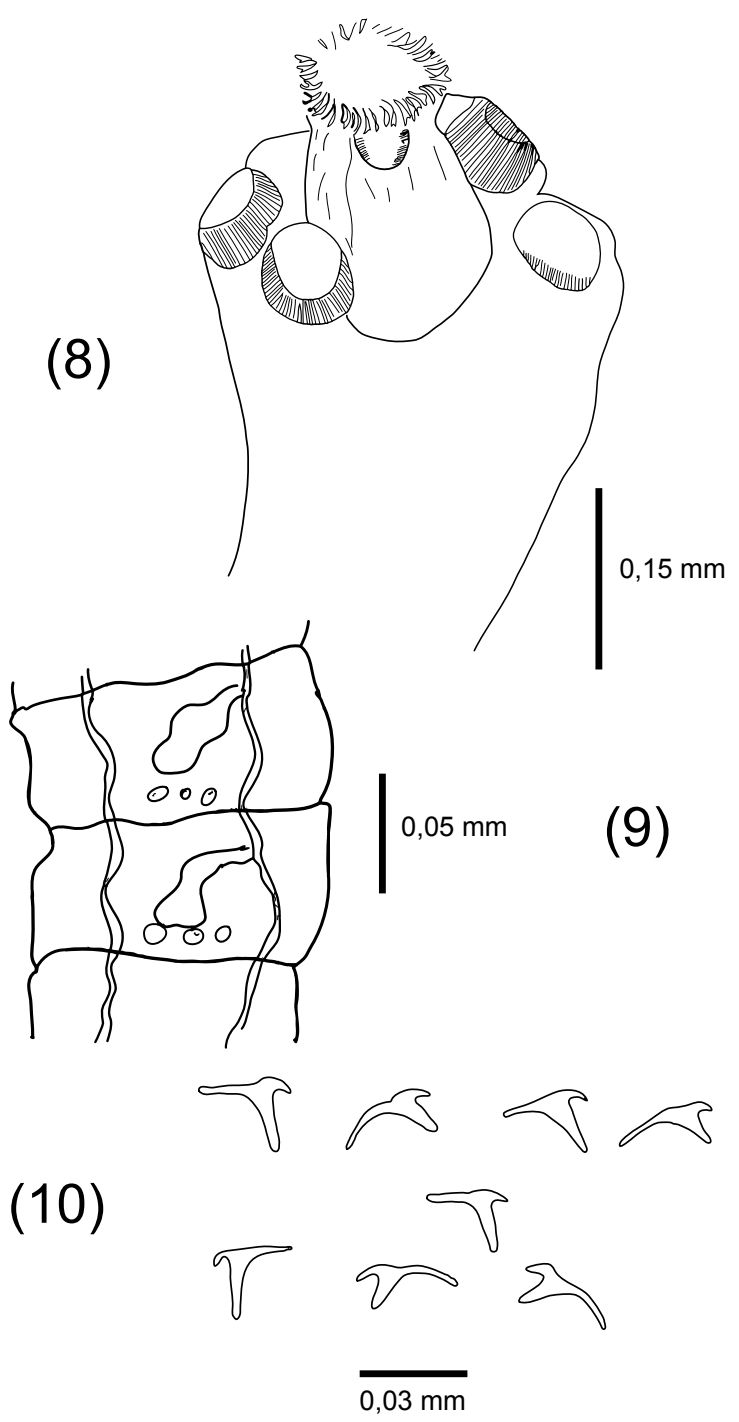

Figura 8-10. Vampirolepis sp. (8) Escolex. (9) Proglótidos en proceso de maduración. (1) Ganchos tipo fraternoide.

diferencias con las 4 especies registradas para el Perú ( $V$. elongatus Rego, 1962, V. phyllostomi Vaucher, 1982, V. mazanensis Vaucher, 1986 y V. artibei Zdzitowiecki \& Rutkoska, 1980) en el número de ganchos; por ejemplo, $V$. mazanensis y $V$. artibei tienen $37-40$ y $22-30$ respectivamente frente a $48-50$ de nuestros especimenes; además, estas dos especies son de mayor tamaño (50 y $32 \mathrm{~mm}$ respectivamente).

Rodentolepis Spasskij, 1954 es un género que se puede confundir con Vampirolepis Spasskij, 1954 porque tienen características comunes y son parásitos de quirópteros; aunque Schmidt (1986) los considera como sinónimos, Jones et al. (1994) indican que la única diferencia fundamental se encuentra en el número de gónadas femeninas que separan a los testículos, siendo dos en Rodentolepis y uno en Vampirolepis.

Esta es la primera vez que se registra a Vampirolepis sp. en Noctilio leporinus y en la zona norte del Perú por lo que se amplía su distribución geográfica.

\section{Agradecimientos}

El presente trabajo fue financiado parcialmente por el proyecto del CSI-UNMSM- N061001021. Agradecemos al Dr. Victor Pacheco, Jefe del departamento de Mastozoología, Museo 
de Historia Natural UNMSM, por su apoyo y ayuda. También agradecemos a Richard Cadenillas por su apoyo (proyectos APECO N 16-2006 y Bat Conservation Internacional, BCI). Finalmente, agradecemos a Liz Huamani, Lisseth Saenz, Mónica Alzamora, Carlos Mendoza, Jael Odar y Juan Carlos Jordán, por su ayuda en la colecta y al Ing. César Peña por su apoyo en la consecución de material bibliográfico.

\section{Literatura citada}

Della-Santa E. 1956. Revisión du genre Oochoristica Lühe (Cestodes). Revue suisse Zool. 63: 1- 113.

Esteban J., L. Oltra, P. Botella y S. Mas. 2006. XI. Helmintos de quirópteros en España: Espectro faunístico e interés aplicado de su estudio. Ministerio de medio ambiente, 1-17. $<$ http:// www.mma.es/secciones/biodiversidad/especies_amenazadas/vertebrados/mamiferos/pdf/CAP11_murciespana_portugal.pdf $>$ (acceso 13/04/08)

Hice C.L., P.M. Velazco \& M.R. Willig. 2004. Bats of the Reserva Nacional Allpahuayo-Mishana, northeastern Peru, with notes on community structure. Acta Chiropterologica 6: 319-334.

Jones A., R.A. Bray \& L.F. Khalil. 1994. Order Cyclophyllidea. In: Khalil, Jones \& Bray (Eds). Keys to the Cestode Parasites of Vertebrates. Wallingford UK: CAB International.
Mendoza L., J. Chavez \& M. Tantaleán. 1997. Cestodos parásitos de murciélagos de Ica, Perú. Parasitol al Dia 21: 20-24.

Pacheco V., R. Cadenillas, S. Velazco, E. Salas \& U. Fajardo. 2007. Noteworthy bat records from the Pacific Tropical rainforest region and adjacent dry forest in northwestern Peru. Acta chiropterologica 9: 409 - 422 .

Simmons N.B \& R.S. VOSS. 1998. The mammals of Paracou, French Guiana: a Neotropical lowland rainforest fauna. Part 1. Bats. Bulletin of the American Museum of Natural History 237: $1-219$.

Schmidt G. 1986. CRC Handbook of tapeworm identification. CRC press, Boca Raton, Florida. 675 p.

Vaucher C. 1982. Helminthes parasites du Paraguay III: Atriotaenia hastati n. sp. (Cestoda: Linstowidae) parasite de Phyllostomus hastatus hastatus (Pallas). Bull. Soc. neuchâtel. Sci. nat. 105: 155-161

Vaucher C. 1986. Cestodes parasites de Chiroptéres en Amérique du Sud II: Hymenolepis mazanensis n.sp., chez Saccopteryx bilineata (Temm.) et Rhinchonycteris naso (Wied - Neuwied), (Chiroptera: Emballonuridae ) en Amazonie péruvienne. Revue suisse zool. 93: 817 - 821. 